\title{
Poles and $\alpha$-points of Meromorphic Solutions of the First Painlevé Hierarchy
}

By

\author{
Shun SHIMOMURA*
}

\begin{abstract}
The first Painlevé hierarchy, which is a sequence of higher order analogues of the first Painleve equation, follows from the singular manifold equations for the $\mathrm{mKdV}$ hierarchy. For meromorphic solutions of the first Painlevé hierarchy, we give a lower estimate for the number of poles; which is regarded as an extension of one corresponding to the first Painlevé equation, and which indicates a conjecture on the growth order. From our main result, two corollaries follow: one is the transcendency of meromorphic solutions, and the other is a lower estimate for the frequency of $\alpha$ points. An essential part of our proof is estimation of certain sums concerning the poles of each meromorphic solution.
\end{abstract}

\section{$\S 1$. Introduction}

For a meromorphic function $f(z)$ in the whole complex plane $\mathbf{C}$, the counting function for poles of $f(z)$ is defined by

$$
N(r, f):=\int_{0}^{r}(n(\rho, f)-n(0, f)) \frac{d \rho}{\rho}+n(0, f) \log r,
$$

where $n(r, f)$ denotes the number of poles inside the disk $|z| \leq r$, each counted according to its multiplicity. Moreover, we use the notation (cf. [6], [8]):

$$
\begin{aligned}
m(r, f) & :=\frac{1}{2 \pi} \int_{0}^{2 \pi} \log ^{+}\left|f\left(r e^{i \phi}\right)\right| d \phi, \quad \log ^{+} x:=\max \{0, \log x\}, \\
T(r, f) & :=m(r, f)+N(r, f)
\end{aligned}
$$

Communicated by T. Kawai. Received May 15, 2003.

2000 Mathematics Subject Classification(s): 34M55, 34M05, 30D35

*Department of Mathematics, Keio University, 3-14-1, Hiyoshi, Kohoku-ku, Yokohama 223-8522, Japan.

e-mail: shimomur@math.keio.ac.jp 
denoting, respectively, the proximity and the characteristic functions; the growth order of $f(z)$ is defined by

$$
\sigma(f):=\limsup _{r \rightarrow \infty} \frac{\log T(r, f)}{\log r} .
$$

Let $w(z)$ be an arbitrary solution of the first Painlevé equation

$$
w^{\prime \prime}=6 w^{2}+z
$$

$\left({ }^{\prime}=d / d z\right)$. Then, $w(z)$ is a transcendental meromorphic function. By a wellknown argument in the Nevanlinna theory, $\lim \sup _{r \rightarrow \infty}(N(r, w) / \log r)=\infty$ (cf. Remark 1.2), which implies that $w(z)$ admits infinitely many poles. This fact is quantitatively represented as

$$
\limsup _{r \rightarrow \infty} \frac{\log N(r, w)}{\log r} \geq \frac{5}{2}
$$

(cf. [11]); and, more precisely,

$$
N(r, w) \gg \frac{r^{5 / 2}}{\log r}
$$

(cf. [5, §7], [13]). These combined with $T(r, w) \ll r^{5 / 2}$ (cf. [5, §8], [12], [14]) imply that the growth order of $w(z)$ is equal to $5 / 2$. (For real-valued functions $\phi(r)$ and $\psi(r)$ on the interval $\left(r_{0},+\infty\right)$, we write $\phi(r) \ll \psi(r)$ or $\psi(r) \gg \phi(r)$ if $\phi(r)=O(\psi(r))$ as $r \rightarrow+\infty$. In the case where $h(z)$ is a function of $z \in \mathbf{C}$, we also write $|h(z)| \ll \psi(r)$ if $|h(z)|=O(\psi(r))$ as $|z|=r \rightarrow+\infty$.)

A sequence of higher order analogues of (PI) is given in the following manner (cf. $[5, \S 16],[7])$. Let $d_{\nu}[w](\nu=0,1,2, \ldots)$ be differential polynomials in $w$ determined by the recursion relation

$$
\begin{aligned}
d_{0}[w] & =1, \\
D d_{\nu+1}[w] & =\left(D^{3}-8 w D-4 w^{\prime}\right) d_{\nu}[w], \quad D=d / d z, \quad \nu \in \mathbf{N} \cup\{0\}
\end{aligned}
$$

(cf. Lemma 2.6 with its proof). Some of them are written in the form

$$
\begin{aligned}
d_{1}[w] / 4 & =-w+C_{10}, \\
d_{2}[w] / 4 & =-w^{\prime \prime}+6 w^{2}+C_{21} d_{1}[w]+C_{20}, \\
d_{3}[w] / 4 & =-w^{(4)}+20 w w^{\prime \prime}+10\left(w^{\prime}\right)^{2}-40 w^{3}+C_{32} d_{2}[w]+C_{31} d_{1}[w]+C_{30}, \\
d_{4}[w] / 4 & =-w^{(6)}+28 w w^{(4)}+56 w^{\prime} w^{(3)}+42\left(w^{\prime \prime}\right)^{2}-280\left(w^{2} w^{\prime \prime}+w\left(w^{\prime}\right)^{2}-w^{4}\right) \\
& +C_{43} d_{3}[w]+C_{42} d_{2}[w]+C_{41} d_{1}[w]+C_{40},
\end{aligned}
$$


where $C_{i j}$ are arbitrary constants. Consider a sequence of $2 \nu$-th order equations of the form

$$
d_{\nu+1}[w]+4 z=0, \quad \nu \in \mathbf{N},
$$

which is called the first Painlevé hierarchy. Equation $\left(\mathrm{PI}_{2}\right)$ essentially coincides with (PI). These equations follow from the singular manifold equations for the mKdV hierarchy (cf. [7], [9], [15]). As in the case of (PI), it is basic and interesting to study analytic properties of meromorphic solutions of $\left(\mathrm{PI}_{2 \nu}\right)$, for example, to determine the growth order of them.

The purpose of this paper is to show the following, which is an extension of (1.1), and which is a first step toward this question:

Theorem 1.1. $\quad$ Suppose that $w_{\nu}(z)$ be a meromorphic solution of $\left(\mathrm{PI}_{2 \nu}\right)$. Then we have

$$
\limsup _{r \rightarrow \infty} \frac{\log N\left(r, w_{\nu}\right)}{\log r} \geq \frac{2 \nu+3}{\nu+1},
$$

namely the growth order of $w_{\nu}(z)$ is not less than $(2 \nu+3) /(\nu+1)$.

Corollary 1.2. Equation $\left(\mathrm{PI}_{2 \nu}\right)$ admits no rational solutions.

Furthermore, the frequency of $\alpha$-points is estimated as follows:

Corollary 1.3. For each $\alpha \in \mathbf{C}$,

$$
\limsup _{r \rightarrow \infty} \frac{\log N\left(r, 1 /\left(w_{\nu}-\alpha\right)\right)}{\log r} \geq \frac{2 \nu+3}{\nu+1} .
$$

Theorem 1.1 with the special case $\nu=1$ leads us to the following:

Conjecture. The growth order of $w_{\nu}(z)$ is equal to $(2 \nu+3) /(\nu+1)$.

Remark 1.1. Since $N\left(r, w_{\nu}\right) \ll n\left(r, w_{\nu}\right) \log r$, the quantity $N\left(r, w_{\nu}\right)$ in (1.5) can be replaced by $n\left(r, w_{\nu}\right)$.

Remark 1.2. For a meromorphic function $f(z)$, the deficiency of $\infty$ is defined by $\delta(\infty, f):=\liminf _{r \rightarrow \infty}(m(r, f) / T(r, f))([6],[8])$. For every solution $w(z)$ of (PI), we have $\delta(\infty, w)=0$; and this fact combined with the transcendency of $w(z)$ implies $\lim \sup _{r \rightarrow \infty}(N(r, w) / \log r)=\infty$ (cf. e.g. [5, §10]). For $w_{\nu}(z)$, analogous deficiency relations are valid: $\delta\left(\infty, w_{\nu}\right)=0$, and for each $\alpha \in \mathbf{C}, \delta\left(\alpha, w_{\nu}\right):=\delta\left(\infty, 1 /\left(w_{\nu}-\alpha\right)\right)=0$ (cf. (3.7) and (4.1) of the proofs of Theorem 1.1 and Corollary 1.3). 
Remark 1.3. The second Painlevé equation

$$
w^{\prime \prime}=2 w^{3}+z w+a, \quad a \in \mathbf{C}
$$

belongs to the second Painlevé hierarchy $\left(\mathrm{PII}_{2 \nu}\right)(\nu \in \mathbf{N})$ (cf. [1], [2], [3], [7]). Value distribution properties of solutions of $\left(\mathrm{PII}_{2 \nu}\right)$ are studied by Gromak and $\mathrm{He}([4])$ and by $\mathrm{Li}$ and $\mathrm{He}([10])$; for example, every transcendental meromorphic solution $w_{\mathrm{II}, \nu}(z)$ satisfies $\delta\left(\infty, w_{\mathrm{II}, \nu}\right)=0$.

Theorem 1.1 and its corollaries are proved in Sections 3 and 4 . In the proofs, we need some basic facts in the Nevanlinna theory and some properties of differential polynomials. They are reviewed or explained in Section 2. To prove Theorem 1.1, we deal with certain sums concerning the poles of each meromorphic solution, which are essential in the proof; and these sums are evaluated in the final section.

\section{$\S 2 . \quad$ Basic Facts}

\section{$\S 2.1$. Nevanlinna Theory}

We review basic facts in the Nevanlinna theory which are necessary in the proofs of our results (cf. [5, Appendix B], [6], [8, Chapters 1 and 2]). Let $f(z)$ be an arbitrary non-constant meromorphic function.

Lemma 2.1. For an arbitrary $\alpha \in \mathbf{C}$,

$$
T(r, 1 /(f-\alpha))=T(r, f)+O(1) .
$$

Lemma 2.2. (i) $T(r, f)$ is a monotone increasing function of $r$.

(ii) $T(r, f) \ll \log r$ if and only if $f(z)$ is a rational function.

(iii) If $f(z)$ is transcendental, then $T(r, f) / \log r \rightarrow \infty$ as $r \rightarrow \infty$.

The following lemmas ([5, Lemmas B.11 and B.12], [8, Lemma 2.4.2 and Proposition 9.2.3]) are useful in the study of differential equations.

Lemma 2.3. Let $f(z)$ be a non-constant meromorphic function satisfying $f^{\lambda+1}=P(z, f)(\lambda \in \mathbf{N})$, where $P(z, u)$ is a polynomial in $z$, $u$ and derivatives of $u$. Suppose that the total degree of $P(z, u)$ with respect to $u$ and its derivatives does not exceed $\lambda$. Then $m(r, f) \ll \log T(r, f)+\log r$ as $r \rightarrow \infty$ outside an exceptional set with finite linear measure.

Lemma 2.4. Let $f(z)$ be a non-constant meromorphic function satisfying $F(z, f)=0$, where $F(z, u)$ is a polynomial in $z$, $u$ and derivatives of $u$. Suppose that $\alpha \in \mathbf{C}$ satisfies $F(z, \alpha) \not \equiv 0$. Then $m(r, 1 /(f-\alpha)) \ll \log T(r, f)+\log r$ as $r \rightarrow \infty$ outside an exceptional set with finite linear measure. 
For real-valued functions, we have ([5, Lemma B.10], [8, Lemma 1,1,1])

Lemma 2.5. Let $\phi(r)$ and $\psi(r)$ be real-valued monotone increasing functions on $(0,+\infty)$. Suppose that $\phi(r) \leq \psi(r)$ outside an exceptional set with finite linear measure. Then $\phi(r) \leq \psi(2 r)$ on $\left(r_{0},+\infty\right)$, where $r_{0}$ is some positive number.

\section{§2.2. Differential Polynomials}

For an arbitrary nonnegative integer $p \in \mathbf{N} \cup\{0\}$, set

$$
\left[w, w^{\prime}, \ldots, w^{(p)}\right]^{\iota}:=\prod_{\kappa=0}^{p}\left(w^{(\kappa)}\right)^{\iota_{\kappa}}, \quad w^{(0)}=w,
$$

where $\iota=\left(\iota_{0}, \iota_{1}, \ldots, \iota_{p}\right) \in(\mathbf{N} \cup\{0\})^{p+1}$. For the index $\iota$, we put

$$
\|\boldsymbol{\iota}\|:=\sum_{\kappa=0}^{p}(\kappa+2) \iota_{\kappa} .
$$

Consider the differential polynomial

$$
\varphi[w]=\sum_{\iota \in I_{\varphi}} c_{\iota}\left[w, w^{\prime}, \ldots, w^{(p)}\right]^{\iota}, \quad c_{\iota} \in \mathbf{C} \backslash\{0\}
$$

where $I_{\varphi} \subset(\mathbf{N} \cup\{0\})^{p+1}$ is a finite set of indices. For $\varphi[w](\not \equiv 0)$, we define its weight by

$$
\operatorname{wt}(\varphi[w])=\max \left\{\|\boldsymbol{\iota}\| \mid \boldsymbol{\iota} \in I_{\varphi}\right\}
$$

in particular, if $\varphi[w] \equiv c_{0} \in \mathbf{C} \backslash\{0\}$, then $\operatorname{wt}\left(c_{0}\right)=0$. For any integer $q>p$, the differential polynomial $\varphi[w]$ admits another expression

$$
\tilde{\varphi}[w]=\sum_{\iota \in I_{\varphi}} c_{\iota}\left[w, w^{\prime}, \ldots, w^{(p)}, \ldots, w^{(q)}\right]^{(\boldsymbol{\iota}, \mathbf{o})}
$$

with $(\boldsymbol{\iota}, \mathbf{o})=\left(\iota_{0}, \iota_{1}, \ldots, \iota_{p}, 0, \ldots, 0\right)$. Then, $\operatorname{wt}(\varphi[w])=\operatorname{wt}(\tilde{\varphi}[w])$, namely the definition of the weight is independent of the choice of the size $q$ of the index. Let $\varphi[w](\not \equiv 0)$ and $\psi[w](\not \equiv 0)$ be arbitrary differential polynomials. Then,

$$
\begin{aligned}
\operatorname{wt}(\varphi[w]+\psi[w]) & =\max \{\operatorname{wt}(\varphi[w]), \operatorname{wt}(\psi[w])\}, \\
\operatorname{wt}(\varphi[w] \psi[w]) & =\operatorname{wt}(\varphi[w])+\operatorname{wt}(\psi[w]) .
\end{aligned}
$$


Remark 2.1. As will be shown later (cf. Lemma 2.7), every pole of $w_{\nu}(z)$ is double. Hence, for every pole of $\left[w_{\nu}, w_{\nu}^{\prime}, \ldots, w_{\nu}^{(2 \nu)}\right]^{\iota}$, the multiplicity of it is equal to $\|\boldsymbol{\iota}\|$. This fact is a background of the definition of the weight of differential polynomials.

We note the following:

Lemma 2.6. For each $\nu \in \mathbf{N} \cup\{0\}, d_{\nu+1}[w]$ is expressible in the form

$$
d_{\nu+1}[w]=\gamma_{\nu+1} w^{\nu+1}+\sum_{\substack{\|\iota\| \leq 2(\nu+1) \\ \iota_{0} \leq \nu}} c_{\iota}\left[w, w^{\prime}, \ldots, w^{(2 \nu)}\right]^{\iota},
$$

where

(i) $\iota=\left(\iota_{0}, \iota_{1}, \ldots, \iota_{2 \nu}\right) \in(\mathbf{N} \cup\{0\})^{2 \nu+1}$,

(ii) $\gamma_{\nu+1} \in \mathbf{C} \backslash\{0\}, c_{\iota} \in \mathbf{C}$.

Proof. By (1.4), for every $\nu \in \mathbf{N}$,

$$
\sum_{\mu=0}^{\nu} d_{\nu-\mu}[w] D d_{\mu+1}[w]=\sum_{\mu=0}^{\nu} d_{\nu-\mu}[w]\left(D^{3}-8 w D-4 w^{\prime}\right) d_{\mu}[w]
$$

and hence, by (1.3),

$$
\begin{aligned}
D d_{\nu+1}[w]=- & \sum_{\mu=0}^{\nu-1} d_{\nu-\mu}[w] D d_{\mu+1}[w] \\
& \quad+\sum_{\mu=0}^{\nu}\left(d_{\nu-\mu}[w] D^{3}-8 w d_{\nu-\mu}[w] D-4 w^{\prime} d_{\nu-\mu}[w]\right) d_{\mu}[w] .
\end{aligned}
$$

Substituting the identities

$$
\begin{aligned}
& \sum_{\mu=0}^{\nu-1} d_{\nu-\mu}[w] D d_{\mu+1}[w]=\frac{1}{2} D\left(\sum_{\mu=0}^{\nu-1} d_{\nu-\mu}[w] d_{\mu+1}[w]\right) \\
& \sum_{\mu=0}^{\nu} d_{\nu-\mu}[w] D^{3} d_{\mu}[w]=D\left(\sum_{\mu=0}^{\nu} d_{\nu-\mu}[w] D^{2} d_{\mu}[w]-\frac{1}{2} \sum_{\mu=0}^{\nu} D d_{\nu-\mu}[w] \cdot D d_{\mu}[w]\right) \\
& w \sum_{\mu=0}^{\nu} d_{\nu-\mu}[w] D d_{\mu}[w]=\frac{1}{2} D\left(w \sum_{\mu=0}^{\nu} d_{\nu-\mu}[w] d_{\mu}[w]\right)-\frac{w^{\prime}}{2} \sum_{\mu=0}^{\nu} d_{\nu-\mu}[w] d_{\mu}[w]
\end{aligned}
$$


we have

$$
\begin{aligned}
D d_{\nu+1}[w]=D & \left(\sum_{\mu=0}^{\nu}\left(d_{\nu-\mu}[w] D^{2}-\frac{1}{2} D d_{\nu-\mu}[w] \cdot D-4 w d_{\nu-\mu}[w]\right) d_{\mu}[w]\right) \\
& -\frac{1}{2} D\left(\sum_{\mu=0}^{\nu-1} d_{\nu-\mu}[w] d_{\mu+1}[w]\right)
\end{aligned}
$$

which implies that

$$
\begin{gathered}
d_{\nu+1}[w]=\sum_{\mu=0}^{\nu}\left(d_{\nu-\mu}[w] D^{2}-\frac{1}{2} D d_{\nu-\mu}[w] \cdot D-4 w d_{\nu-\mu}[w]\right) d_{\mu}[w] \\
-\frac{1}{2} \sum_{\mu=0}^{\nu-1} d_{\nu-\mu}[w] d_{\mu+1}[w]+C_{\nu}
\end{gathered}
$$

where $C_{\nu}$ is an arbitrary constant. By $(2.2)$ combined with $(1.3), d_{\nu+1}[w]$ is a differential polynomial. Moreover, in $d_{\nu+1}[w]$, the derivative with the highest order is $w^{(2 \nu)}$. Indeed, this fact is inductively checked by using (1.3) and (1.4). Hence, for every $\nu \in \mathbf{N} \cup\{0\}, d_{\nu+1}[w]$ is written in the form

$$
d_{\nu+1}[w]=\sum_{\iota \in I_{\nu}} \tilde{c}_{\iota}\left[w, w^{\prime}, \ldots, w^{(2 \nu)}\right]^{\iota}, \quad \iota=\left(\iota_{0}, \iota_{1}, \ldots, \iota_{2 \nu}\right), \quad \tilde{c}_{\iota} \in \mathbf{C} \backslash\{0\},
$$

where $I_{\nu} \subset(\mathbf{N} \cup\{0\})^{2 \nu+1}$ is a finite set of indices. We prove (2.1) by induction on $\nu$. Clearly it is valid for $\nu=0$. Suppose that (2.1) is valid for every $\nu \leq N$; namely, $\operatorname{wt}\left(d_{\nu+1}[w]\right) \leq 2(\nu+1)$ for every $\nu \leq N$, and $\gamma_{N+1} \in \mathbf{C} \backslash\{0\}$. Since, for $\iota \neq \mathbf{o}$,

$$
\begin{aligned}
& \operatorname{wt}\left(D\left(\left[w, w^{\prime}, \ldots, w^{(2 \nu)}\right]^{\iota}\right)\right)=\operatorname{wt}\left(\left[w, w^{\prime}, \ldots, w^{(2 \nu)}\right]^{\iota} \sum_{\mu=0}^{2 \nu} \iota_{\mu} w^{(\mu+1)} / w^{(\mu)}\right) \\
& =\max \left\{\operatorname{wt}\left(w^{\iota_{0}}\left(w^{\prime}\right)^{\iota_{1}} \cdots\left(w^{(2 \nu)}\right)^{\iota_{2 \nu}} w^{(\mu+1)} / w^{(\mu)}\right) \mid 0 \leq \mu \leq 2 \nu, \iota_{\mu} \neq 0\right\} \\
& =\max \left\{\|\boldsymbol{\iota}\|+(\mu+2)(-1)+(\mu+3) \cdot 1 \mid 0 \leq \mu \leq 2 \nu, \iota_{\mu} \neq 0\right\}=\|\boldsymbol{\iota}\|+1 \\
& =\operatorname{wt}\left(\left[w, w^{\prime}, \ldots, w^{(2 \nu)}\right]^{\iota}\right)+1,
\end{aligned}
$$

we have $\operatorname{wt}\left(D^{l} d_{\nu+1}[w]\right)=l+\operatorname{wt}\left(d_{\nu+1}[w]\right) \leq l+2(\nu+1)$ for $\nu \leq N$ and for $l=1,2$. Hence, by $(2.2)$ with $\nu=N+1$, wt $\left(d_{N+2}[w]\right) \leq 2(N+1)+2=2(N+2)$. Furthermore, by (1.4),

$$
\begin{aligned}
D d_{N+2}[w] & =-8 w D\left(\gamma_{N+1} w^{N+1}\right)-4 w^{\prime} \cdot \gamma_{N+1} w^{N+1}+\cdots \\
& =-4 \gamma_{N+1}(2 N+3) w^{N+1} w^{\prime}+\cdots
\end{aligned}
$$


which implies that $\gamma_{N+2}=-4(2 N+3)(N+2)^{-1} \gamma_{N+1} \in \mathbf{C} \backslash\{0\}$. Hence, (2.1) is valid for $\nu=N+1$ as well. This completes the proof.

Lemma 2.7. For a meromorphic solution $w_{\nu}(z)$ of $\left(\mathrm{PI}_{2 \nu}\right)$, let $a_{0}$ be an arbitrary pole of it. Then, around $z=a_{0}$,

$$
w_{\nu}(z)=c\left(a_{0}\right)\left(z-a_{0}\right)^{-2}+O(1),
$$

where $c\left(a_{0}\right)=k\left(a_{0}\right)\left(k\left(a_{0}\right)+1\right) / 2$ for some integer $k\left(a_{0}\right) \in\{1, \ldots, \nu\}$.

Proof. Around the pole $z=a_{0}$, we write $w_{\nu}(z)=b\left(z-a_{0}\right)^{-\sigma}+\cdots$, $b \neq 0$. Suppose that $\sigma \geq 3$. It is inductively shown that $d_{k}\left[w_{\nu}\right](z)=$ $b_{k}\left(z-a_{0}\right)^{-\sigma k}+\cdots, b_{k} \neq 0$ for every $k \in \mathbf{N}$, because this formula with $k$ implies

$$
\begin{aligned}
D d_{k+1}\left[w_{\nu}\right](z) & =\left(D^{3}-8 w_{\nu}(z) D-4 w_{\nu}^{\prime}(z)\right) d_{k}\left[w_{\nu}\right](z) \\
& =(-8(-\sigma k)-4(-\sigma)) b b_{k}\left(z-a_{0}\right)^{-\sigma(k+1)-1}+\cdots \\
& =4(2 k+1) \sigma b b_{k}\left(z-a_{0}\right)^{-\sigma(k+1)-1}+\cdots,
\end{aligned}
$$

namely

$$
d_{k+1}\left[w_{\nu}\right](z)=-4(2 k+1)(k+1)^{-1} b b_{k}\left(z-a_{0}\right)^{-\sigma(k+1)}+\cdots .
$$

Hence, if $\sigma \geq 3$, substitution of $d_{\nu+1}\left[w_{\nu}\right](z)=b_{\nu+1}\left(z-a_{0}\right)^{-\sigma(\nu+1)}+\cdots$ into $\left(\mathrm{PI}_{2 \nu}\right)$ yields a contradiction. Supposing that $\sigma=1$, by an analogous argument, we can show that $d_{\nu+1}\left[w_{\nu}\right](z)=b_{\nu+1}^{\prime}\left(z-a_{0}\right)^{-(2 \nu+1)}+\cdots, b_{\nu+1}^{\prime} \neq 0$, and also derive a contradiction. Therefore, $z=a_{0}$ is a double pole. Put $w_{\nu}(z)=$ $b_{0}\left(z-a_{0}\right)^{-2}+\cdots, b_{0} \neq 0$. Since substitution of $d_{k}\left[w_{\nu}\right](z)=A_{k}\left(z-a_{0}\right)^{-2 k}+\cdots$, $k \in \mathbf{N}$ into (1.4) yields that $d_{k+1}\left[w_{\nu}\right](z)=A_{k+1}\left(z-a_{0}\right)^{-2(k+1)}+\cdots$ with

$$
A_{k+1}=-4(2 k+1)(k+1)^{-1}\left(b_{0}-k(k+1) / 2\right) A_{k} .
$$

By this fact,

$$
A_{\nu+1}=B_{\nu+1} b_{0} \prod_{k=1}^{\nu}\left(b_{0}-k(k+1) / 2\right), \quad B_{\nu+1} \neq 0 .
$$

Substituting $d_{\nu+1}\left[w_{\nu}\right](z)$ into $\left(\mathrm{PI}_{2 \nu}\right)$, we have $b_{0}=k(k+1) / 2$ for some $k \in$ $\{1, \ldots, \nu\}$. Furthermore, the relation

$$
\begin{aligned}
D d_{\nu+2}\left[w_{\nu}\right](z) & =\left(D^{3}-8 w_{\nu}(z) D-4 w_{\nu}^{\prime}(z)\right) d_{\nu+1}\left[w_{\nu}\right](z) \\
& =\left(D^{3}-8 w_{\nu}(z) D-4 w_{\nu}^{\prime}(z)\right)(-4 z)=32 w_{\nu}(z)+16 z w_{\nu}^{\prime}(z) \\
& =16 D\left(z w_{\nu}(z)\right)+16 w_{\nu}(z),
\end{aligned}
$$


namely

$$
16 w_{\nu}(z)=D\left(d_{\nu+2}\left[w_{\nu}\right](z)-16 z w_{\nu}(z)\right)
$$

means that the residue of $w_{\nu}(z)$ at the pole $z=a_{0}$ vanishes. This completes the proof.

\section{$\S 3 . \quad$ Proof of Theorem 1.1}

To prove (1.5), we suppose the contrary:

$$
\limsup _{r \rightarrow \infty} \frac{\log N\left(r, w_{\nu}\right)}{\log r}<\frac{2 \nu+3}{\nu+1}
$$

namely, for some $\varepsilon>0, N\left(r, w_{\nu}\right) \ll r^{(2 \nu+3) /(\nu+1)-\varepsilon}$, from which it follows that

$$
n(r)=n\left(r, w_{\nu}\right) \ll r^{(2 \nu+3) /(\nu+1)-\varepsilon},
$$

because

$$
N\left(2 r, w_{\nu}\right) \geq \int_{r}^{2 r}\left(n\left(\rho, w_{\nu}\right)-n\left(0, w_{\nu}\right)\right) \frac{d \rho}{\rho} \geq\left(n\left(r, w_{\nu}\right)+O(1)\right) \log 2 .
$$

Starting from (3.1), we would like to derive a contradiction. Let $\left\{a_{j}\right\}_{j \in J}$ be a sequence of all distinct poles of $w_{\nu}(z)$ arranged as $\left|a_{1}\right| \leq \cdots \leq\left|a_{j}\right| \leq \cdots$, where $J=\mathbf{N}$ or $\{1, \ldots, p\}(p \in \mathbf{N})$ or $\emptyset$. Clearly these poles do not accumulate at any point in C. By Lemma 2.7, we write $w_{\nu}(z)$ in the form

$$
\begin{aligned}
& w_{\nu}(z)=\Phi(z)+g(z), \\
& \Phi(z)=\sum_{j \in J} c\left(a_{j}\right)\left(\left(z-a_{j}\right)^{-2}-a_{j}^{-2}\right),
\end{aligned}
$$

where $g(z)$ is an entire function. In (3.4), we make the following conventions: (i) if $a_{1}=0$, then the term $\left(z-a_{1}\right)^{-2}-a_{1}^{-2}$ is replaced by $z^{-2}$; (ii) if $J=\emptyset$, then $\Phi(z) \equiv 0$. In what follows, we may suppose that $\Phi(z) \not \equiv 0$, because the case where $\Phi(z) \equiv 0$ is similarly treated by adding a slight modification. Under (3.2), we have the following lemmas whose proofs will be given afterward:

Lemma 3.1. For every $r>1$, there exists $z_{r}$ satisfying $0.7 r \leq\left|z_{r}\right| \leq r$,

$$
\sum_{\left|a_{j}\right|<2 r}\left|z_{r}-a_{j}\right|^{-2} \ll r^{1 /(\nu+1)-\varepsilon / 2}, \quad \sum_{\left|a_{j}\right|<2 r}\left|z_{r}-a_{j}\right|^{-3} \ll r^{(3 / 2) /(\nu+1)-\varepsilon} .
$$


Lemma 3.2. Let $r$ be an arbitrary number satisfying $r>1$. Then,

$$
\sum_{\left|a_{j}\right| \geq 2 r}\left|\left(z-a_{j}\right)^{-2}-a_{j}^{-2}\right| \ll r^{1 /(\nu+1)-\varepsilon}, \quad \sum_{\left|a_{j}\right| \geq 2 r}\left|z-a_{j}\right|^{-3} \ll 1
$$

for $|z| \leq r$, and

$$
\sum_{0<\left|a_{j}\right|<2 r}\left|a_{j}^{-2}\right| \ll r^{1 /(\nu+1)-\varepsilon} .
$$

Lemma 3.3. $\quad$ There exists a set $E \subset(0, \infty)$ with finite linear measure such that

$$
\sum_{0<\left|a_{j}\right|<\infty}\left|\left(z-a_{j}\right)^{-2}-a_{j}^{-2}\right| \ll|z|^{9} \quad \text { for }|z| \in(0, \infty) \backslash E .
$$

By Lemma 2.6, $w_{\nu}(z)$ satisfies the equation

$$
-\gamma_{\nu+1} w^{\nu+1}=\sum_{\|\iota\| \leq 2(\nu+1)} c_{\iota} w^{\iota_{0}}\left(w^{\prime}\right)^{\iota_{1}} \cdots\left(w^{(2 \nu)}\right)^{\iota_{2} \nu}+4 z .
$$

For each term on the right-hand side, note that

$$
\sum_{\kappa=0}^{2 \nu} \iota_{\kappa} \leq \nu
$$

because $2 \sum_{\kappa=0}^{2 \nu} \iota_{\kappa}=\|\boldsymbol{\iota}\|-\sum_{\kappa=0}^{2 \nu} \kappa \iota_{\kappa}=2(\nu+1)$ is valid if and only if $\iota=$ $\left(\iota_{0}, \iota_{1}, \ldots, \iota_{2 \nu}\right)=(\nu+1,0, \ldots, 0)$. By Lemma 2.3 , there exists a set $E^{*} \subset(0, \infty)$ with finite linear measure such that

$$
m\left(r, w_{\nu}\right) \ll \log T\left(r, w_{\nu}\right)+\log r
$$

as $r \rightarrow \infty, r \notin E^{*}$. (Note that $\left(\mathrm{PI}_{2 \nu}\right)$ does not admit a constant solution.) Combining this with $(3.1)$, we have $T\left(r, w_{\nu}\right) \ll r^{(2 \nu+3) /(\nu+1)}$ and $m\left(r, w_{\nu}\right) \ll$ $\log r$ for $r \notin E^{*}$. By Lemma 3.3, for $r \notin E \cup E^{*}$,

$$
T(r, g)=m(r, g)=m\left(r, w_{\nu}-\Phi\right) \leq m\left(r, w_{\nu}\right)+m(r, \Phi) \ll \log r .
$$

By Lemmas 2.5 and 2.2, this is valid for $r$ approaching $\infty$ without an exceptional set, and hence $g(z)$ is a polynomial.

By Lemmas 3.1 and 3.2, for every $r>1$, there exists $z_{r}, 0.7 r \leq\left|z_{r}\right| \leq r$ satisfying

$$
\begin{aligned}
\left|\Phi\left(z_{r}\right)\right| \ll & \sum_{\left|a_{j}\right|<2 r}\left|z_{r}-a_{j}\right|^{-2}+\sum_{0<\left|a_{j}\right|<2 r}\left|a_{j}^{-2}\right| \\
& +\sum_{\left|a_{j}\right| \geq 2 r}\left|\left(z_{r}-a_{j}\right)^{-2}-a_{j}^{-2}\right| \ll r^{1 /(\nu+1)-\varepsilon / 2} .
\end{aligned}
$$


Then, also for every $\kappa=1,2, \ldots, 2 \nu$,

$$
\left|\Phi^{(\kappa)}\left(z_{r}\right)\right| \ll r^{(\kappa / 2+1) /(\nu+1)-\varepsilon} .
$$

Indeed, observing that

$$
\left|\Phi^{(\kappa)}\left(z_{r}\right)\right| \ll \sum_{\left|a_{j}\right|<2 r}\left|z_{r}-a_{j}\right|^{-2-\kappa}+\sum_{\left|a_{j}\right| \geq 2 r}\left|z_{r}-a_{j}\right|^{-2-\kappa},
$$

we have the following:

(i) if $\kappa$ is odd,

$$
\begin{aligned}
\left|\Phi^{(\kappa)}\left(z_{r}\right)\right| \ll & \left(\sum_{\left|a_{j}\right|<2 r}\left|z_{r}-a_{j}\right|^{-3}\right)\left(\sum_{\left|a_{j}\right|<2 r}\left|z_{r}-a_{j}\right|^{-2}\right)^{(\kappa-1) / 2} \\
& \quad+\sum_{\left|a_{j}\right| \geq 2 r}\left|z_{r}-a_{j}\right|^{-3} \\
& \ll r^{(3 / 2) /(\nu+1)-\varepsilon} r^{(1 /(\nu+1)-\varepsilon / 2)(\kappa-1) / 2} \\
& \ll r^{(\kappa / 2+1) /(\nu+1)-\varepsilon}
\end{aligned}
$$

(ii) if $\kappa$ is even,

$$
\begin{aligned}
\left|\Phi^{(\kappa)}\left(z_{r}\right)\right| & \ll\left(\sum_{\left|a_{j}\right|<2 r}\left|z_{r}-a_{j}\right|^{-2}\right)^{\kappa / 2+1}+\sum_{\left|a_{j}\right| \geq 2 r}\left|z_{r}-a_{j}\right|^{-3} \\
& \ll r^{(1 /(\nu+1)-\varepsilon / 2)(\kappa / 2+1)} \ll r^{(\kappa / 2+1) /(\nu+1)-\varepsilon}
\end{aligned}
$$

From (3.5), we have

$$
\left|w_{\nu}\left(z_{r}\right)\right| \ll\left(\left|z_{r}\right|+\sum_{\substack{\|\iota\| \leq 2(\nu+1) \\ \iota_{0} \leq \nu}}\left|w_{\nu}\left(z_{r}\right)^{\iota_{0}} w_{\nu}^{\prime}\left(z_{r}\right)^{\iota_{1}} \cdots w_{\nu}^{(2 \nu)}\left(z_{r}\right)^{\iota_{2 \nu}}\right|\right)^{1 /(\nu+1)} .
$$

Now suppose that $\operatorname{deg} g(z)=\delta_{0} \geq 1$. Substitute $w_{\nu}^{(\kappa)}\left(z_{r}\right)=g^{(\kappa)}\left(z_{r}\right)+$ $\Phi^{(\kappa)}\left(z_{r}\right)(\kappa=0,1, \ldots, 2 \nu)$ into (3.10), and observe that

$$
\left|w_{\nu}\left(z_{r}\right)\right| \geq\left|g\left(z_{r}\right)\right|-\left|\Phi\left(z_{r}\right)\right|=\left|g\left(z_{r}\right)\right|+O\left(r^{1 /(\nu+1)}\right) \gg r^{\delta_{0}},
$$

and that, for every $\iota$ satisfying $\iota_{0} \leq \nu$ and $\|\iota\| \leq 2(\nu+1)$,

$$
\begin{aligned}
& \left|w_{\nu}\left(z_{r}\right)^{\iota_{0}} w_{\nu}^{\prime}\left(z_{r}\right)^{\iota_{1}} \cdots w_{\nu}^{(2 \nu)}\left(z_{r}\right)^{\iota_{2 \nu}}\right| \\
& \ll\left(\left|g\left(z_{r}\right)\right|+\left|\Phi\left(z_{r}\right)\right|\right)^{\iota_{0}}\left(\left|g\left(z_{r}\right)\right|+\left|\Phi^{\prime}\left(z_{r}\right)\right|\right)^{\iota_{1}} \cdots\left(\left|g\left(z_{r}\right)\right|+\left|\Phi^{(2 \nu)}\left(z_{r}\right)\right|\right)^{\iota_{2 \nu}} \\
& \ll\left|z_{r}\right|^{\delta_{0}\left(\iota_{0}+\iota_{1}+\cdots+\iota_{2 \nu}\right)} \ll r^{\delta_{0} \nu}
\end{aligned}
$$


(cf. (3.6), (3.8) and (3.9)). Then, we have the contradiction $r^{\delta_{0}} \ll$ $\left(\left|z_{r}\right|+r^{\delta_{0} \nu}\right)^{1 /(\nu+1)} \ll r^{\delta_{0} \nu /(\nu+1)}$; which implies that $g(z) \equiv C \in \mathbf{C}$. Substituting $w=w_{\nu}(z)=\Phi(z)+C$ into $\left(\mathrm{PI}_{2 \nu}\right)$, and observing that, for every $\iota$ satisfying $0<\|\boldsymbol{\iota}\| \leq 2(\nu+1)$,

$$
\begin{aligned}
& \left|w_{\nu}\left(z_{r}\right)^{\iota_{0}} w_{\nu}^{\prime}\left(z_{r}\right)^{\iota_{1}} \cdots w_{\nu}^{(2 \nu)}\left(z_{r}\right)^{\iota_{2 \nu}}\right| \\
& \ll\left(\left|\Phi\left(z_{r}\right)\right|+|C|\right)^{\iota_{0}}\left|\Phi^{\prime}\left(z_{r}\right)\right|^{\iota_{1}} \cdots\left|\Phi^{(2 \nu)}\left(z_{r}\right)\right|^{\iota_{2 \nu}} \ll r^{\chi(\iota)-\varepsilon / 2}
\end{aligned}
$$

with $\chi(\iota)=\sum_{\kappa=0}^{2 \nu}(\kappa / 2+1) \iota_{\kappa} /(\nu+1)=(\|\iota\| / 2) /(\nu+1) \leq 1$ (cf. (3.8) and $(3.9))$, we have

$$
0.7 r \leq\left|z_{r}\right| \ll\left|d_{\nu+1}\left[w_{\nu}\right]\left(z_{r}\right)\right| \ll r^{1-\varepsilon / 2}
$$

which is a contradiction. We have thus proved (1.5).

\section{$\S 4 . \quad$ Proofs of Corollaries 1.2 and 1.3}

Corollary 1.2 immediately follows from Theorem 1.1. To prove Corollary 1.3 , note that $w \equiv \alpha(\in \mathbf{C})$ is not a solution of $\left(\mathrm{PI}_{2 \nu}\right)$. By Lemma 2.4,

$$
m\left(r, 1 /\left(w_{\nu}-\alpha\right)\right) \ll \log T\left(r, w_{\nu}\right)+\log r
$$

as $r \rightarrow \infty$ for $r \notin E_{1}$, where $E_{1} \subset(0, \infty)$ is a set with finite linear measure. Since $w_{\nu}(z)$ is transcendental, by Lemmas 2.1 and 2.2, we have

$$
\frac{N\left(r, 1 /\left(w_{\nu}-\alpha\right)\right)}{T\left(r, w_{\nu}\right)}=1-\frac{m\left(r, 1 /\left(w_{\nu}-\alpha\right)\right)+O(1)}{T\left(r, w_{\nu}\right)} \rightarrow 1 \quad \text { as } r \rightarrow \infty, r \notin E_{1} ;
$$

and hence

$$
N\left(r, 1 /\left(w_{\nu}-\alpha\right)\right) \geq(1 / 2) T\left(r, w_{\nu}\right) \quad \text { for } r \in\left(r_{1}, \infty\right) \backslash E_{1},
$$

for some $r_{1}>0$. On the other hand, by (3.7), $N\left(r, w_{\nu}\right) / T\left(r, w_{\nu}\right) \rightarrow 1$ as $r \rightarrow \infty$, $r \notin E^{*}$. Hence,

$$
(1 / 2) N\left(r, w_{\nu}\right) \leq T\left(r, w_{\nu}\right) \quad \text { for } r \in\left(r_{2}, \infty\right) \backslash E^{*},
$$

for some $r_{2}>0$. Using Lemma 2.5, from (4.2) and (4.3), we derive that $N\left(r, w_{\nu}\right) \leq 4 N\left(2 r, 1 /\left(w_{\nu}-\alpha\right)\right)$ for $r \in\left(r_{3}, \infty\right)$, where $r_{3}>0$ is sufficiently large. This inequality combined with (1.5) yields the conclusion (1.6) of Corollary 1.3 . 


\section{§5. Proofs of Lemmas 3.1, 3.2 and 3.3}

\section{$\S 5.1$. Proof of Lemma 3.1}

Put $D_{r}=\{z|| z \mid \leq r\}$ and $\Delta_{0}=\mathbf{C} \backslash\left(\bigcup_{j \geq 1} U_{j}\right)$; where $U_{j}=\left\{z|| z-a_{j} \mid<\right.$ $\left.\left|a_{j}\right|^{-(1 / 2) /(\nu+1)}\right\}$ if $a_{j} \neq 0$, and $U_{1}=\{z|| z \mid<1\}$ if $a_{1}=0$. Since, by (3.2),

$$
\begin{aligned}
& \sum_{1<\left|a_{j}\right|<r}\left|a_{j}\right|^{-1 /(\nu+1)}=\int_{1}^{r} \rho^{-1 /(\nu+1)} d n(\rho) \\
&=\left[\rho^{-1 /(\nu+1)} n(\rho)\right]_{1}^{r}+\frac{1}{\nu+1} \int_{1}^{r} \rho^{-1-1 /(\nu+1)} n(\rho) d \rho \ll r^{2-\varepsilon}
\end{aligned}
$$

we can take $r_{0}$ so large that $7 \pi r^{2} / 8 \leq \mu\left(\Delta_{0} \cap D_{r}\right)<\pi r^{2}$ for every $r>r_{0}$, where $\mu(X)$ denotes the area of a set $X$. For every $r>1$, if $\left|a_{j}\right|<2 r$, then

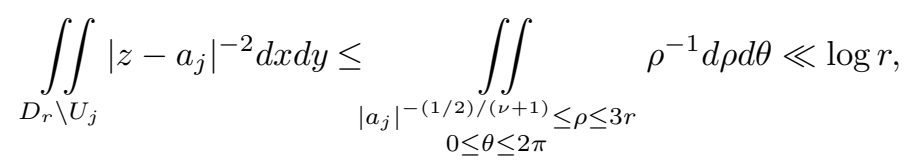

and

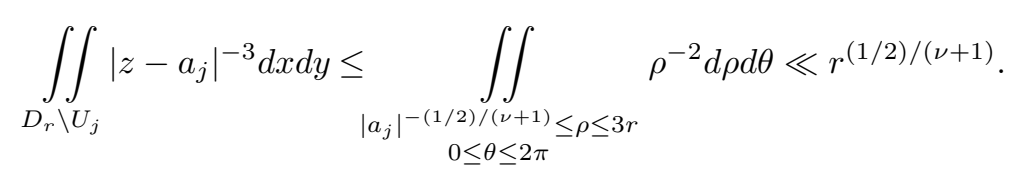

Hence,

$$
\iint_{\Delta_{0} \cap D_{r}} \sum_{\left|a_{j}\right|<2 r}\left|z-a_{j}\right|^{-2} d x d y \ll n(2 r) \log r \leq K_{0} r^{(2 \nu+3) /(\nu+1)-\varepsilon / 2},
$$

$$
\iint_{\Delta_{0} \cap D_{r}} \sum_{\left|a_{j}\right|<2 r}\left|z-a_{j}\right|^{-3} d x d y \ll n(2 r) r^{(1 / 2) /(\nu+1)} \leq K_{0} r^{2+(3 / 2) /(\nu+1)-\varepsilon},
$$

where $K_{0}$ is some positive number. Consider the sets

$$
\begin{aligned}
& F_{r}^{1}=\left\{z \in \Delta_{0} \cap D_{r}\left|\sum_{\left|a_{j}\right|<2 r}\right| z-\left.a_{j}\right|^{-2} \leq 8 \pi^{-1} K_{0} r^{1 /(\nu+1)-\varepsilon / 2}\right\}, \\
& F_{r}^{2}=\left\{z \in \Delta_{0} \cap D_{r}\left|\sum_{\left|a_{j}\right|<2 r}\right| z-\left.a_{j}\right|^{-3} \leq 8 \pi^{-1} K_{0} r^{(3 / 2) /(\nu+1)-\varepsilon}\right\} .
\end{aligned}
$$

Suppose that $\mu\left(F_{r}^{1}\right)<3 \pi r^{2} / 4$. Then

$$
\begin{aligned}
\iint_{\Delta_{0} \cap D_{r} \backslash F_{r}^{1}} \sum_{\left|a_{j}\right|<2 r}\left|z-a_{j}\right|^{-2} d x d y & >8 \pi^{-1} K_{0} r^{1 /(\nu+1)-\varepsilon / 2}(7 / 8-3 / 4) \pi r^{2} \\
& =K_{0} r^{(2 \nu+3) /(\nu+1)-\varepsilon / 2}
\end{aligned}
$$


which contradicts (5.1). This implies that $\mu\left(F_{r}^{1}\right) \geq 3 \pi r^{2} / 4$. By the same argument, we have $\mu\left(F_{r}^{2}\right) \geq 3 \pi r^{2} / 4$. Hence, $\mu\left(F_{r}^{1} \cap F_{r}^{2}\right) \geq \pi r^{2} / 2$. Observing that $\mu(\{z|| z \mid<0.7 r\})=0.49 \pi r^{2}$, we have $\{z|0.7 r \leq| z \mid \leq r\} \cap\left(F_{r}^{1} \cap F_{r}^{2}\right) \neq \emptyset$, which implies the conclusion.

\section{$\S 5.2$. Proof of Lemma 3.2}

For $\left|a_{j}\right| \geq 2 r$, and for $z \in D_{r}$, observing that $\left|z / a_{j}\right| \leq 1 / 2$, we have $\left|z-a_{j}\right|^{-3} \leq 8\left|a_{j}\right|^{-3}$, and

$$
\left|\left(z-a_{j}\right)^{-2}-a_{j}^{-2}\right|=2|z|\left|a_{j}\right|^{-3}\left|1-\left(z / a_{j}\right) / 2\right|\left|1-z / a_{j}\right|^{-2} \leq 10 r\left|a_{j}\right|^{-3} .
$$

Hence, by (3.2),

$$
\begin{aligned}
& \sum_{\left|a_{j}\right| \geq 2 r}\left|\left(z-a_{j}\right)^{-2}-a_{j}^{-2}\right| \ll r \sum_{\left|a_{j}\right| \geq 2 r}\left|a_{j}\right|^{-3} \ll r \int_{2 r}^{\infty} t^{-3} d n(t) \\
& \ll r \int_{2 r}^{\infty} t^{-4} n(t) d t \ll r^{1 /(\nu+1)-\varepsilon}, \\
& \sum_{\left|a_{j}\right| \geq 2 r}\left|z-a_{j}\right|^{-3} \ll \sum_{\left|a_{j}\right| \geq 2 r}\left|a_{j}\right|^{-3} \ll \int_{2 r}^{\infty} t^{-3} d n(t) \ll 1,
\end{aligned}
$$

and

$$
\begin{aligned}
\sum_{0<\left|a_{j}\right|<2 r}\left|a_{j}^{-2}\right| & \ll \int_{1}^{2 r} t^{-2} d n(t)+O(1) \\
& \ll r^{1 /(\nu+1)-\varepsilon}+\int_{1}^{2 r} t^{-3} n(t) d t+O(1) \ll r^{1 /(\nu+1)-\varepsilon} .
\end{aligned}
$$

Thus the lemma is proved.

\section{§5.3. Proof of Lemma 3.3}

We put

$$
E=\left(0,\left|a_{1}\right|+1\right) \cup\left(\bigcup_{j \in J \backslash\{1\}}\left(\left|a_{j}\right|-\left|a_{j}\right|^{-3},\left|a_{j}\right|+\left|a_{j}\right|^{-3}\right)\right) .
$$

Since, by (3.2),

$$
\sum_{j \in J \backslash\{1\}}\left|a_{j}\right|^{-3} \ll \int_{1}^{\infty} t^{-3} d n(t)+O(1) \ll \int_{1}^{\infty} t^{-4} n(t) d t+O(1) \ll 1,
$$


the total length of $E$ is finite. If $|z| \notin E$, then

$$
\begin{aligned}
\left(\sum_{0<\left|a_{j}\right|<2|z|}+\sum_{\left|a_{j}\right| \geq 2|z|}\right) & \left|\left(z-a_{j}\right)^{-2}-a_{j}^{-2}\right| \\
& \ll\left(|z|^{6}+1\right) n(2|z|)+|z|^{1 /(\nu+1)} \ll|z|^{9} .
\end{aligned}
$$

This completes the proof.

\section{References}

[1] Airault, H., Rational solutions of Painlevé equations, Stud. Appl. Math., 61 (1979), 31-53.

[2] Flaschka, H. and Newell, A. C., Monodromy- and spectrum-preserving deformations, I, Commun. Math. Phys., 76 (1980), 65-116.

[3] Gromak, V. I., Nonlinear evolution equations and equations of P-type, Differential Equations, 20 (1984), 1435-1439.

[4] Gromak, V. I. and He, Y., On the solutions of the second Painlevé equation of higher order, Proc. Math. Inst. Belarus Natl. Acad. Sci., 4 (2000), 37-48.

[5] Gromak, V. I., Laine, I. and Shimomura, S., Painlevé Differential Equations in the Complex Plane, Walter de Gruyter, Berlin, New York, 2002.

[6] Hayman, W. K., Meromorphic Functions, Clarendon Press, Oxford, 1964.

[7] Kudryashov, N. A., The first and second Painlevé equations of higher order and some relations between them, Phys. Lett. A, 224 (1997), 353-360.

[8] Laine, I., Nevanlinna Theory and Complex Differential Equations, Walter de Gruyter, Berlin, New York, 1993.

[9] Lax, P. D., Almost periodic solutions of the KdV equation, SIAM Rev., 18 (1976), 351-375.

[10] Li, Y. and He, Y., On analytic properties of higher analogs of the second Painlevé equation, J. Math. Phys., 43 (2002), 1106-1115.

[11] Mues, E. and Redheffer, R., On the growth of logarithmic derivatives, J. London Math. Soc., 8 (1974), 412-425.

[12] Shimomura, S., Growth of the first, the second and the fourth Painlevé transcendents, Math. Proc. Cambridge Philos. Soc., 134 (2003), 259-269.

[13] , Lower estimates for the growth of Painlevé transcendents, Funkcial. Ekvac., 46 (2003), 287-295.

[14] Steinmetz, N., Value distribution of Painlevé transcendents, Israel J. Math., 128 (2002), 29-52.

[15] Weiss, J., On classes of integrable systems and the Painlevé property, J. Math. Phys., 25 (1984), 13-24. 